\title{
THE EMBEDDING THEOREM \\ FOR THE BESOV AND TRIEBEL-LIZORKIN SPACES ON SPACES OF HOMOGENEOUS TYPE
}

\author{
Y.-S. HAN
}

(Communicated by J. Marshall Ash)

\begin{abstract}
In this note the classical embedding theorem for the Besov and Triebel-Lizorkin spaces on $R^{n}$ is generalized to the Besov and Triebel-Lizorkin spaces on spaces of homogeneous type. The proof is new even for $R^{n}$ case.
\end{abstract}

\section{INTRODUCTION}

Suppose a function $\phi$ satisfies the conditions: (i) $\phi \in S$; (ii) Supp $\hat{\phi} \subseteq$ $\left\{\xi \in R^{n}: \frac{1}{2} \leq|\xi| \leq 2\right\}$; (iii) $|\hat{\phi}(\xi)| \geq c>0$ if $\frac{3}{5} \leq|\xi| \leq \frac{5}{3}$. The Besov and Triebel-Lizorkin spaces can be defined as follows:

$$
\|f\|_{\dot{B}_{p}^{\alpha, q}}=\left\{f \in S^{\prime} / P:\left\{\sum_{k \in Z}\left(2^{k \alpha}\left\|\phi_{k} * f\right\|_{p}\right)^{q}\right\}^{1 / q}<\infty\right\}
$$

for $0<p, q \leq \infty, \alpha \in R$, and

$$
\|f\|_{\dot{F}_{p}^{\alpha, q}}=\left\{f \in S^{\prime} / P:\left\|\left\{\sum_{k \in Z}\left(2^{k \alpha}\left|\phi_{k} * f\right|\right)^{q}\right\}^{1 / q}\right\|_{p}<\infty\right\}
$$

for $0<p<\infty, 0<q \leq \infty$, and $\alpha \in R$, where $P$ is the collection of all polynomials and $\phi_{k}(x)=2^{k n} \phi\left(2^{k} x\right)$.

The classical embedding theorem for these spaces is given by the following.

Theorem A. Suppose $-\infty<s_{1}<s_{0}<\infty, 0<p_{0}, p_{1}<\infty, 0<q_{0}, q_{1}, q \leq \infty$, and $s_{0}-\frac{n}{p_{0}}=s_{1}-\frac{n}{p_{1}}$. Then

$$
\begin{aligned}
& \dot{B}_{p_{0}}^{s_{0}, q} \rightarrow \dot{B}_{p_{1}}^{s_{1}, q}, \\
& \dot{F}_{p_{0}}^{s_{0}, q_{0}} \rightarrow \dot{F}_{p_{1}}^{s_{1}, q_{1}} .
\end{aligned}
$$

Received by the editors March 31, 1993 and, in revised form, November 15, 1993.

1991 Mathematics Subject Classification. Primary 42B25, 46E35.

Key words and phrases. Embedding theorem, Besov and Triebel-Lizorkin spaces, spaces of homogeneous type. 
We remark that since $\dot{F}_{p}^{0,2}=L^{p}$ for $1<p<\infty$ and $\dot{F}_{p}^{\alpha, 2}=I_{\alpha}\left(L^{p}\right)$, where $I_{\alpha}, 0<\alpha<n$, is the Riesz potential, the theorem above includes the classical Sobolev embedding theorem. See [P] and [T] for more details.

In this note we will generalize the classical embedding theorem for the Besov and Triebel-Lizorkin spaces on $R^{n}$ to the Besov and Triebel-Lizorkin spaces on spaces of homogeneous type in the sense of Coifman and Weiss ([CW]).

We begin by recalling the definitions necessary for introducing the Besov and Triebel-Lizorkin spaces on spaces of homogeneous type. A quasi-metric $d$ on a set $X$ is a function $d: X \times X \rightarrow[0, \infty]$ satisfying:

(i) $\quad d(x, y)=0$ if and only if $x=y$,

(ii) $d(x, y)=d(y, x)$ for all $x, y \in X$,

(iii) there exists a constant $A<\infty$ such that for all $x, y, z \in X$,

$$
d(x, y) \leq A[d(x, z)+d(z, y)] \text {. }
$$

Any quasi-metric defines a topology, for which the balls $B(x, r)=\{y \in$ $X: d(y, x)<r\}$ form a base. However, the balls themselves need not be open when $A>1$.

Definition 1.2 ([CW]). A space of homogeneous type $(X, d, \mu)$ is a set $X$ together with a quasi-metric $d$ and a nonnegative measure $\mu$ on $X$ such that $\mu(B(x, r))<\infty$ for all $x \in X$ and all $r>0$, and there exists $A^{\prime}<\infty$ such that for all $x \in X$ and all $r>0$,

$$
\mu(B(x, 2 r)) \leq A^{\prime} \mu(B(x, r)) .
$$

Here $\mu$ is assumed to be defined on a $\sigma$-algebra which contains all Borel sets and all balls $B(x, r)$.

Macias and Segovia [MS] have shown that one can replace $d$ by another quasi-metric $\rho$ such that there exist $c<\infty$ and some $\theta, 0<\theta<1$,

$$
\begin{gathered}
\rho(x, y) \approx \inf \{\mu(B): B \text { is a ball containing } x \text { and } y\}, \\
\left|\rho(x, y)-\rho\left(x^{\prime}, y\right)\right| \leq c \rho\left(x, x^{\prime}\right)^{\theta}\left[\rho(x, y)+\rho\left(x^{\prime}, y\right)\right]^{1-\theta} \\
\text { for all } x, x^{\prime}, \text { and } y \in X .
\end{gathered}
$$

There are many interesting examples of spaces of homogeneous type. For instance, any $C^{\infty}$ compact Riemannian manifold with the Riemannian metric and volume and the boundary of any bounded Lipschitz domain in $R^{n}$ with the induced Euclidean metric and the Lebesgue measure are spaces of homogeneous type. See [Ch] for more examples. The regularity exponent $\theta$ depends on spaces of homogeneous type, for example, $\theta=1$ for any bounded Lipschitz domain in $R^{n}$. We will suppose that $\mu(X)=\infty$ and $\mu(\{x\})=0$ for all $x \in X$. These hypotheses allow us to construct an approximation to the identity (see [HS]).

Definition 1.6. A sequence $\left(S_{k}\right)_{k \in Z}$ of operators is called to be an approximation to the identity if $S_{k}(x, y)$, the kernels of $S_{k}$, are functions from $X \times X$ into $\mathscr{C}$ such that there exists a constant $C$, some $0<\varepsilon \leq \theta$, and some $c<\infty$, for all $k \in Z$ and all $x, x^{\prime}, y$, and $y^{\prime} \in X$,

$$
\begin{gathered}
S_{k}(x, y)=0 \text { if } \rho(x, y) \geq c 2^{-k} \text { and }\left\|S_{k}\right\|_{\infty} \leq C 2^{k}, \\
\left|S_{k}(x, y)-S_{k}\left(x^{\prime}, y\right)\right| \leq C 2^{k(1+\varepsilon)} \rho\left(x, x^{\prime}\right)^{\varepsilon}
\end{gathered}
$$




$$
\left|S_{k}(x, y)-S_{k}\left(x, y^{\prime}\right)\right| \leq C 2^{k(1+\varepsilon)} \rho\left(y, y^{\prime}\right)^{\varepsilon},
$$

(iv)

$\left|\left[S_{k}(x, y)-S_{k}\left(x, y^{\prime}\right)\right]-\left[S_{k}\left(x^{\prime}, y\right)-S_{k}\left(x^{\prime}, y^{\prime}\right)\right]\right| \leq C \rho\left(x, x^{\prime}\right)^{\varepsilon} \rho\left(y, y^{\prime}\right)^{\varepsilon} 2^{k(1+2 \varepsilon)}$,

$$
\begin{aligned}
& \int_{X} S_{k}(x, y) d \mu(y)=1, \\
& \int_{X} S_{k}(x, y) d \mu(x)=1 .
\end{aligned}
$$

See [DJS] for the existence of such a sequence of operators; there all conditions are introduced and checked except condition (iv) in 1.6. It is easy to see that the same construction in [DJS] satisfies condition (iv). To define the Besov and Triebel-Lizorkin spaces on spaces of homogeneous type we need the following definition (see [HS]).

Definition 1.7. Fix two exponents $0<\beta \leq \theta$ and $\gamma>0$. A function $f$ defined on $X$ is said to be a strong smooth molecule of type $(\beta, \gamma)$ centered at $x_{0} \in X$ with width $d>0$, if $f$ satisfies the following conditions:

$$
\begin{gathered}
|f(x)| \leq c \frac{d^{\gamma}}{\left(d+\rho\left(x, x_{0}\right)\right)^{1+\gamma}}, \\
\left|f(x)-f\left(x^{\prime}\right)\right| \leq c\left[\frac{\rho\left(x, x^{\prime}\right)}{d+\rho\left(x, x_{0}\right)}\right]^{\beta} \frac{d^{\gamma}}{\left(d+\rho\left(x, x_{0}\right)\right)^{1+\gamma}}
\end{gathered}
$$

for $\rho\left(x, x^{\prime}\right) \leq \frac{1}{2 A}\left(d+\rho\left(x, x_{0}\right)\right)$,

$$
\int_{X} f(x) d \mu(x)=0 .
$$

This definition was first introduced in [M] for the case $X=R^{n}$ with condition (ii) in (1.7) replaced by

$$
\left|f(x)-f\left(x^{\prime}\right)\right| \leq c\left[\frac{\rho\left(x, x^{\prime}\right)}{d}\right]^{\beta}\left[\frac{d^{\gamma}}{\left(d+\rho\left(x, x_{0}\right)\right)^{1+\gamma}}+\frac{d^{\gamma}}{\left(d+\rho\left(x^{\prime}, x_{0}\right)\right)^{1+\gamma}}\right] .
$$

The collection of all strong smooth molecules of type $(\beta, \gamma)$ centered at $x_{0} \in$ $X$ with width $d>0$ will be denoted by $\mathscr{M}^{(\beta, \gamma)}\left(x_{0}, d\right)$. If $f \in \mathscr{M}^{(\beta, \gamma)}\left(x_{0}, d\right)$, the norm of $f$ in $\mathscr{M}^{(\beta, \gamma)}\left(x_{0}, d\right)$ is then defined by

$$
f \|_{\mathscr{K}^{(\beta, \gamma)}\left(x_{0}, d\right)}=\inf \{c \geq 0 \text { : (i) and (ii) in (1.7) hold }\} .
$$

Now we fix a point $x_{0} \in X$ and denote the class of all $f \in \mathscr{M}^{(\beta, \gamma)}\left(x_{0}, 1\right)$ by $\mathscr{M}^{(\beta, \gamma)}$. It is easy to see that $\mathscr{M}^{(\beta, \gamma)}$ is a Banach space under the norm $\|f\|_{\mathscr{R}^{(\beta, \gamma)}}<\infty$. Just as the space of distributions $\mathscr{S}^{\prime}$ is defined on $R^{n}$, the dual space $\left(\mathscr{M}^{(\beta, \gamma)}\right)^{\prime}$ consists of all linear functionals $\mathscr{L}$ from $\mathscr{M}^{(\beta, \gamma)}$ to $\mathscr{C}$ with the property that there exists a finite constant $c$ such that for all

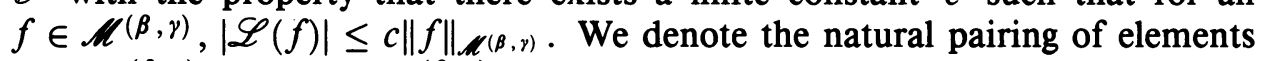
$h \in\left(\mathscr{M}^{(\beta, \gamma)}\right)^{\prime}$ and $f \in \mathscr{M}^{(\beta, \gamma)}$ by $\langle h, f\rangle$. It is also easy to see that for $x_{1} \in X$ and $d>0, \mathscr{M}^{(\beta, \gamma)}\left(x_{1}, d\right)=\mathscr{M}^{(\beta, \gamma)}$ with equivalent norms. Thus, $\langle h, f\rangle$ is well defined for all $h \in\left(\mathscr{M}^{(\beta, \gamma)}\right)^{\prime}$ and all $f \in \mathscr{M}^{(\beta, \gamma)}\left(x_{1}, d\right)$ with $x_{1} \in X$ and 
$d>0$. In [HS] the Besov and Triebel-Lizorkin spaces on spaces of homogeneous type were introduced by use of the sequence of operators $\left(D_{k}\right)_{k \in Z}$ where $D_{k}(f)(x)=\int_{X} D_{k}(x, y) f(y) d \mu(y)$ and $D_{k}(x, y)=S_{k}(x, y)-S_{k-1}(x, y)$ and $\left(S_{k}\right)_{k \in Z}$ is the approximation to the identity defined in (1.6). More precisely, the Besov space $\dot{B}_{p}^{\alpha, q}$ for $-\varepsilon<\alpha<\varepsilon$ and $1 \leq p, q \leq \infty$ is the collection of all $f \in\left(\mathscr{M}^{(\beta, \gamma)}\right)^{\prime}$ with $0<\beta, \gamma<\varepsilon$ such that

$$
\|f\|_{\dot{B}_{p}^{\alpha, q}}=\left\{\sum_{k \in Z}\left(2^{k \alpha}\left\|D_{k}(f)\right\|_{p}\right)^{q}\right\}^{1 / q}<\infty .
$$

The Triebel-Lizorkin space $\dot{F}_{p}^{\alpha, q}$ for $-\varepsilon<\alpha<\varepsilon$ and $1<p, q<\infty$ is the collection of all $f \in\left(\mathscr{M}^{(\beta, \gamma)}\right)^{\prime}$ with $0<\beta, \gamma<\varepsilon$ such that

$$
\|f\|_{\dot{F}_{p}^{\alpha, q}}=\left\|\left\{\sum_{k \in Z}\left(2^{k \alpha}\left|D_{k}(f)\right|\right)^{q}\right\}^{1 / q}\right\|_{p}<\infty .
$$

In this note we prove the following embedding theorem for the Besov and Triebel-Lizorkin spaces on spaces of homogeneous type.

Theorem. Suppose $-\varepsilon<s_{1}<s_{0}<\varepsilon$. Then

(i) $\dot{B}_{p_{0}}^{s_{0}, q} \rightarrow \dot{B}_{p_{1}}^{s_{1}, q}$ for $1 \leq q \leq \infty, 1 \leq p_{0}, p_{1} \leq \infty$, and $-\varepsilon<s_{0}-\frac{1}{p_{0}}=$ $s_{1}-\frac{1}{p_{1}}<\varepsilon$ $\varepsilon$.

(ii) $\dot{F}_{p_{0}}^{s_{0}, q_{0}} \rightarrow \dot{F}_{p_{1}}^{s_{1}}, q_{1}$ for $1<p_{0}, p_{1}, q_{0}, q_{1}<\infty$, and $-\varepsilon<s_{0}-\frac{1}{p_{0}}=s_{1}-\frac{1}{p_{1}}<$

\section{Proof of The Theorem}

The proof of the classical embedding Theorem A depends on the Fourier transform. To be precise, if $\phi$ is a function as in the definition of the Besov and Triebel-Lizorkin spaces and $f \in \mathscr{S}^{\prime} / \mathscr{P}$, then, using the Fourier transform, we have the inequality

$$
\left\|\phi_{k} * f\right\|_{\infty} \leq c 2^{k n / p}\left\|\phi_{k} * f\right\|_{p}
$$

which, by Hölder's inequality, yields

$$
\left\|\phi_{k} * f\right\|_{p_{1}} \leq c 2^{k n\left(1 / p_{0}-1 / p_{1}\right)}\left\|\phi_{k} * f\right\|_{p_{0}} .
$$

This gives (i) of Theorem A. Similarly, the proof of (ii) of Theorem A also needs (2.1). Since there is no Fourier transform on spaces of homogeneous type, we need a new idea to prove the Theorem. Our starting point is to use the Calderon type reproducing formula which was obtained in [HS]. Since we never use the Fourier transform, our method is new even for the case of $R^{n}$.

The Calderon type reproducing formula ([HS]). Suppose $\left\{D_{k}\right\}$ is the family of operators used in the definitions of the Besov and Triebel-Lizorkin spaces. Then there exists a sequence of operators $\left\{\widetilde{D}_{k}\right\}$ such that for all $f \in\left(\mathscr{M}^{(\beta, \gamma)}\right)^{\prime}$

$$
f=\sum_{k} \widetilde{D}_{k} D_{k}(f)
$$




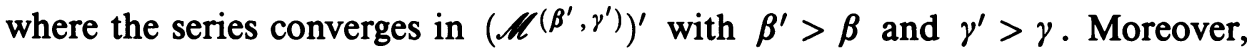
$\widetilde{D}_{k}(x, y)$, the kernel of $\widetilde{D}_{k}$, satisfies the following estimates: For $0<\varepsilon^{\prime}<\varepsilon$ there exists a constant $c$ such that

$$
\left|\widetilde{D}_{k}(x, y)\right| \leq c \frac{2^{-k \varepsilon^{\prime}}}{\left(2^{-k}+\rho(x, y)\right)^{1+\varepsilon^{\prime}}},
$$

$$
\left|\widetilde{D}_{k}(x, y)-\widetilde{D}_{k}\left(x^{\prime}, y\right)\right| \leq c\left[\frac{\rho\left(x, x^{\prime}\right)}{\left(2^{-k}+\rho(x, y)\right)}\right]^{e^{\prime}} \frac{2^{-k \varepsilon^{\prime}}}{\left(2^{-k}+\rho(x, y)\right)^{1+\varepsilon^{\prime}}}
$$

for $\rho\left(x, x^{\prime}\right) \leq \frac{1}{2 A}\left(2^{-k}+\rho(x, y)\right)$,

$$
\int \widetilde{D}_{k}(x, y) d \mu(x)=0 \text { for all } y \in X .
$$

Lemma 2.4. For $0<\varepsilon^{\prime}<\varepsilon$ there exists a constant $c$ such that $D_{k} \widetilde{D}_{j}(x, y)$, the kernel of $D_{k} \widetilde{D}_{j}$, satisfies the following estimate:

$$
\left|D_{k} \widetilde{D}_{j}(x, y)\right| \leq c 2^{-|k-j| \varepsilon^{\prime}} \frac{\left(2^{-k} \vee 2^{-j}\right)^{\varepsilon^{\prime}}}{\left\{\left(2^{-k} \vee 2^{-j}\right)+\rho(x, y)\right\}^{1+\varepsilon^{\prime}}}
$$

where $a \vee b$ denotes the maximum of $a$ and $b$.

We now prove (2.5). Notice that the kernel of $D_{k}$ satisfies the conditions (i)-(iv) in (1.6) and $\int D_{k}(x, y) d \mu(y)=0$, and $\int D_{k}(x, y) d \mu(x)=0$. Consider first that $k \geq j$ and $2 c A 2^{-k} \leq 2^{-j}$ or $k \geq j$ and $\rho(x, y) \geq 2 c A 2^{-j}$. Then

$$
\begin{aligned}
\left|D_{k} \widetilde{D}_{j}(x, y)\right| & =\left|\int D_{k}(x, z) \widetilde{D}_{j}(z, y) d \mu(z)\right| \\
& =\left|\int D_{k}(x, z)\left[\widetilde{D}_{j}(z, y)-\widetilde{D}_{j}(x, y)\right] d \mu(z)\right|
\end{aligned}
$$

since $\int D_{k}(x, z) d \mu(z)=0$

$$
\leq c \int\left|D_{k}(x, z)\right| \frac{\rho(x, z)^{e^{\prime}}}{\left(2^{-j}+\rho(x, y)\right)^{\varepsilon^{\prime}}} \frac{2^{-j e^{\prime}}}{\left\{2^{-j}+\rho(x, y)\right\}^{1+\varepsilon^{\prime}}} d \mu(z)
$$

by the fact that $\rho(x, z) \leq c 2^{-k} \leq \frac{1}{2 A}\left(2^{-j}+\rho(x, y)\right)$ and the smoothness of the kernel of $\widetilde{D}_{j}$

$$
\leq c \frac{2^{-k \varepsilon^{\prime}}}{\left\{2^{-j}+\rho(x, y)\right\}^{1+\varepsilon^{\prime}}}
$$

by the size condition of the kernel of $D_{k}$ in (i) of (1.6)

$$
\leq c 2^{-(k-j) \varepsilon^{\prime}} \frac{2^{-j \varepsilon^{\prime}}}{\left\{2^{-j}+\rho(x, y)\right\}^{1+\varepsilon^{\prime}}}
$$

which shows (2.5) for the case where $k \geq j$ and $2 c A 2^{-k} \leq 2^{-j}$ or $k \geq j$, and $\rho(x, y) \geq 2 c A 2^{-j}$.

Consider now the case that $k \geq j, 2 c A 2^{-k}>2^{-j}$ and $\rho(x, y)<2 c A 2^{-j}$. Then

$$
\left|D_{k} \widetilde{D}_{j}(x, y)\right|=\left|\int D_{k}(x, z) \widetilde{D}_{j}(z, y) d \mu(z)\right| \leq c 2^{j}
$$


by the size conditions on the kernel of $D_{k}$ and $\widetilde{D}_{j}$

$$
\leq c 2^{(j-k) \varepsilon^{\prime}} \frac{2^{-j \varepsilon^{\prime}}}{\left\{2^{-j}+\rho(x, y)\right\}^{1+\varepsilon^{\prime}}}
$$

by the facts that $2^{(k-j) \varepsilon^{\prime}} \leq(2 c A)^{\varepsilon^{\prime}}$ and $\rho(x, y)<2 c A 2^{-j}$, which together with the above estimate shows (2.5) for the case $k \geq j$. The proof of $(2.5)$ for the case $k<j$ is similar and easier.

We may assume that $\varepsilon^{\prime}$, the regularity exponent in the Calderon type reproducing formula, satisfies $-\varepsilon^{\prime}<s_{1}<s_{0}<\varepsilon^{\prime}$ and $-\varepsilon^{\prime}<s_{0}-\frac{1}{p_{0}}=s_{1}-\frac{1}{p_{1}}<\varepsilon^{\prime}$. Now we prove (i) of the Theorem. Suppose that $f \in \dot{B}_{p_{0}}^{s_{0}, q}$. By the Calderon type reproducing formula we have

$$
\begin{aligned}
\left\|D_{k}(f)\right\|_{p_{1}} & =\left\|\sum_{j} D_{k} \widetilde{D}_{j} D_{j}(f)\right\|_{p_{1}} \leq \sum_{j}\left\|D_{k} \widetilde{D}_{j} D_{j}(f)\right\|_{p_{1}} \\
& \leq c \sum_{j} 2^{-|k-j| \varepsilon^{\prime}}\left(2^{-k} \vee 2^{-j}\right)^{\left(1 / p_{1}-1 / p_{0}\right)}\left\|D_{j}(f)\right\|_{p_{0}}
\end{aligned}
$$

by Young's inequality and (2.5). Thus,

$$
\begin{aligned}
\|f\|_{B_{p_{1}}^{s_{1}, q}}= & \left\{\sum_{k \in Z}\left(2^{k s_{1}}\left\|D_{k}(f)\right\|_{p_{1}}\right)^{q}\right\}^{1 / q} \\
\leq & c\left\{\sum_{k \in Z}\left(2^{k s_{1}} \sum_{j} 2^{-|k-j| \varepsilon^{\prime}}\left(2^{-k} \vee 2^{-j}\right)^{\left(1 / p_{1}-1 / p_{0}\right)}\left\|D_{j}(f)\right\|_{p_{0}}\right)^{q}\right\}^{1 / q} \\
\leq & c\left\{\sum_{k \in Z}\left(\sum_{j>k} 2^{-|k-j| \varepsilon^{\prime}} 2^{k s_{1}} 2^{-k\left(1 / p_{1}-1 / p_{0}\right)}\left\|D_{j}(f)\right\| p_{0}\right)^{q}\right\}^{1 / q} \\
& +c\left\{\sum_{k \in Z}\left(\sum_{j \leq k} 2^{-|k-j| \varepsilon^{\prime}} 2^{k s_{1}} 2^{-j\left(1 / p_{1}-1 / p_{0}\right)}\left\|D_{j}(f)\right\|_{p_{0}}\right)^{q}\right\}^{1 / q} \\
\leq & c\left\{\sum_{k \in Z}\left(\sum_{j>k} 2^{-|k-j| \varepsilon^{\prime}} 2^{k s_{0}}\left\|D_{j}(f)\right\|_{p_{0}}\right)^{q}\right\}^{1 / q} \\
& +c\left\{\sum_{k \in Z}\left(\sum_{j \leq k} 2^{-|k-j| \varepsilon^{\prime}} 2^{k s_{1}} 2^{-j\left(s_{1}-s_{0}\right)}\left\|D_{j}(f)\right\|_{p_{0}}\right)^{q}\right\}^{1 / q}
\end{aligned}
$$

by the fact that $\frac{1}{p_{1}}-\frac{1}{p_{0}}=s_{1}-s_{0}$

$$
\leq c\left\{\sum_{j \in Z}\left(2^{j s_{0}}\left\|D_{j}(f)\right\|_{p_{0}}\right)^{q}\right\}^{1 / q}
$$


by Hölder's inequality and the fact that

$$
\begin{aligned}
& \sum_{k \in Z} 2^{-|k-j| \varepsilon^{\prime}} 2^{(k-j) s_{0}}+\sum_{k \in Z} 2^{-|k-j| \varepsilon^{\prime}} 2^{(k-j) s_{1}} \\
& +\sum_{j \in Z} 2^{-|k-j| \varepsilon^{\prime}} 2^{(k-j) s_{0}}+\sum_{j \in Z} 2^{-|k-j| \varepsilon^{\prime}} 2^{(k-j) s_{1}}<\infty
\end{aligned}
$$

if $-\varepsilon^{\prime}<s_{1}<s_{0}<\varepsilon^{\prime}$

$$
=c\|f\|_{\dot{B}_{p_{0}}^{s_{0}, q}} .
$$

To prove (ii) of the Theorem, by the homogeneity, it suffices to take $\|f\|_{\dot{F}_{p_{0}}^{s_{0}, q_{0}}}$ $=1$. By the Calderon type reproducing formula, Hölder's inequality, and the estimate in (2.5),

$$
\begin{aligned}
\left|D_{k}(f)\right| & =\left|\sum_{j} D_{k} \widetilde{D}_{j} D_{j}(f)\right| \leq \sum_{j}\left|D_{k} \widetilde{D}_{j} D_{j}(f)\right| \\
& \leq c \sum_{j} 2^{-|k-j| \varepsilon^{\prime}}\left(2^{-k} \vee 2^{-j}\right)^{-1 / p_{0}}\left\|D_{j}(f)\right\|_{p_{0}} \\
& \leq c \sum_{j} 2^{-|k-j| \varepsilon^{\prime}}\left(2^{-k} \vee 2^{-j}\right)^{-1 / p_{0}} 2^{-j s_{0}}\left\|\left\{\sum_{k \in Z}\left(2^{k s_{0}}\left|D_{k}(f)\right|\right)^{q_{0}}\right\}^{1 / q_{0}}\right\| \\
& \leq c \sum_{j} 2^{-|k-j| \varepsilon^{\prime}}\left(2^{k} \vee 2^{j}\right)^{1 / p_{0}} 2^{-j s_{0}} .
\end{aligned}
$$

Therefore, for any fixed integer $N$

$$
\begin{aligned}
& \left\{\sum_{-\infty}^{N}\left(2^{k s_{1}}\left|D_{k}(f)\right|\right)^{q_{1}}\right\}^{1 / q_{1}} \\
& \leq c\left\{\sum_{-\infty}^{N}\left(2^{k s_{1}} \sum_{j} 2^{-|k-j| \varepsilon^{\prime}}\left(2^{k} \vee 2^{j}\right)^{1 / p_{0}} 2^{-j s_{0}}\right)^{q_{1}}\right\}^{1 / q_{1}} \\
& \left.\left.\leq c\left\{\sum_{-\infty}^{N}\left(2^{k s_{1}} \sum_{j>k} 2^{-|k-j| \varepsilon^{\prime}} 2^{j / p_{0}} 2^{-j s_{0}}\right)^{q_{1}}\right\}^{1 / q_{1}}\right\}^{q_{1}}\right\}^{1 / q_{1}} \\
& +\left\{\sum_{-\infty}^{N}\left(2^{k s_{1}} \sum_{j \leq k} 2^{-|k-j| \varepsilon^{\prime}} 2^{k / p_{0}} 2^{-j s_{0}}\right\}^{1 / q_{1}}\right. \\
& \leq c\left\{\sum_{-\infty}^{N}\left(2^{k / p_{1}}\right)^{q_{1}}\right\}^{1}
\end{aligned}
$$

since $s_{0}-\frac{1}{p_{0}}=s_{1}-\frac{1}{p_{1}}$ and $\sum_{j>k} 2^{-|k-j| \varepsilon^{\prime}} 2^{(j-k)\left(1 / p_{0}-s_{0}\right)}+\sum_{j \leq k} 2^{-|k-j| \varepsilon^{\prime}} 2^{(k-j) s_{0}}<$ 
2188

Y.-S. HAN

$\infty$ if $-\varepsilon^{\prime}+\frac{1}{p_{0}}<s_{0}<\varepsilon^{\prime} \leq c 2^{N / p_{1}}$. On the other hand,

$$
\begin{aligned}
& \left\{\sum_{N}^{\infty}\left(2^{k s_{1}}\left|D_{k}(f)\right|\right)^{q_{1}}\right\}^{1 / q_{1}}=\left\{\sum_{N}^{\infty}\left(2^{k\left(s_{1}-s_{0}\right)} 2^{k s_{0}}\left|D_{k}(f)\right|\right)^{q_{1}}\right\}^{1 / q_{1}} \\
& \leq\left\{\sum_{N}^{\infty}\left(2^{k\left(s_{1}-s_{0}\right)}\right)^{q_{1}}\right\}^{1 / q_{1}}\left\{\sum_{k \in Z}\left(2^{k s_{0}}\left|D_{k}(f)\right|\right)^{q_{0}}\right\}^{1 / q_{0}} \\
& \leq c 2^{N\left(s_{1}-s_{0}\right)}\left\{\sum_{k \in Z}\left(2^{k s_{0}}\left|D_{k}(f)\right|\right)^{q_{0}}\right\}^{1 / q_{0}}
\end{aligned}
$$

since $s_{1}<s_{0}$

$$
\leq c 2^{N\left(1 / p_{1}-1 / p_{0}\right)}\left\{\sum_{k \in Z}\left(2^{k s_{0}}\left|D_{k}(f)\right|\right)^{q_{0}}\right\}^{1 / q_{0}}
$$

since $s_{0}-1 / p_{0}=s_{1}-1 / p_{1}$. We now obtain

$$
\begin{aligned}
&\|f\|_{F_{p_{1}}^{s_{1}, q_{1}}}^{p_{1}}=p_{1} \int_{0}^{\infty} t^{p_{1}-1}\left|\left\{\left(\sum_{k \in Z}\left(2^{k s_{1}}\left|D_{k}(f)\right|\right)^{q_{1}}\right)^{1 / q_{1}}>t\right\}\right| d t \\
& \leq p_{1} \sum_{-\infty}^{\infty} \int_{2 c 2^{N / p_{1}}}^{2 c^{(N+1) / p_{1}}} t^{p_{1}-1}\left|\left\{\left(\sum_{k \in Z}\left(2^{k s_{1}}\left|D_{k}(f)\right|\right)^{q_{1}}\right)^{1 / q_{1}}>t\right\}\right| d t \\
& \leq p_{1} \sum_{-\infty}^{\infty} \int_{2 c 2^{N / p_{1}}}^{2 c 2^{(N+1) / p_{1}}} t^{p_{1}-1} \mid\left\{\left(\sum_{-\infty}^{N}\left(2^{k s_{1}}\left|D_{k}(f)\right|\right)^{q_{1}}\right)^{1 / q_{1}}\left|\left\{\left(\sum_{N}^{\infty}\left(2^{k s_{1}}\left|D_{k}(f)\right|\right)^{q_{1}}\right)^{1 / q_{1}}>t\right\}\right| d t\right. \\
& \leq p_{1} \sum_{-\infty}^{\infty} \int_{2 c 2^{N / p_{1}}}^{2 c 2^{(N+1) / p_{1}}} t^{p_{1}-1}\left|\left\{\left(\sum_{N}^{\infty}\left(2^{k s_{1}}\left|D_{k}(f)\right|\right)^{q_{1}}\right)^{1 / q_{1}}>\frac{1}{2} t\right\}\right| d t
\end{aligned}
$$

by (2.7)

$$
\leq p_{1} \sum_{-\infty}^{\infty} \int_{2 c 2^{N / p_{1}}}^{2 c 2^{(N+1) / p_{1}}} t^{p_{1}-1}\left|\left\{\left(\sum_{-\infty}^{\infty}\left(2^{k s_{0}}\left|D_{k}(f)\right|\right)^{q_{0}}\right)^{1 / q_{0}}>\frac{1}{2} c 2^{N\left(1 / p_{0}-1 / p_{1}\right)} t\right\}\right| d t
$$

by $(2.8)$

$$
\leq p_{1} \sum_{-\infty}^{\infty} \int_{2 c 2^{N / p_{1}}}^{2 c 2^{(N+1) / p_{1}}} t^{p_{1}-1}\left|\left\{\left(\sum_{-\infty}^{\infty}\left(2^{k s_{0}}\left|D_{k}(f)\right|\right)^{q_{0}}\right)^{1 / q_{0}}>c t^{p_{1} / p_{0}}\right\}\right| d t
$$


since $t \approx 2^{N / p_{1}}$

$$
\begin{aligned}
& \leq p_{1} \int_{0}^{\infty} t^{p_{1}-1}\left|\left\{\left(\sum_{-\infty}^{\infty}\left(2^{k s_{0}}\left|D_{k}(f)\right|\right)^{q_{0}}\right)^{1 / q_{0}}>c t^{p_{1} / p_{0}}\right\}\right| d t \\
& \leq c p_{1} \int_{0}^{\infty} t^{p_{0}-1}\left|\left\{\left(\sum_{-\infty}^{\infty}\left(2^{k s_{0}}\left|D_{k}(f)\right|\right)^{q_{0}}\right)^{1 / q_{0}}>c t\right\}\right| d t \\
& \leq c\|f\|_{\dot{F}_{p_{0}}^{p_{0}, q_{0}}}^{p_{0}} \leq c .
\end{aligned}
$$

\section{REFERENCES}

[CW] R. Coifman and G. Weiss, Analyse harmonique noncommutative sur certains espaces, Lecture Notes in Math., vol. 242, Springer-Verlag, Berlin, Heidelberg, and New York, 1971.

[Ch] M. Christ, Singular integral operators, NSF-CBMS Regional Conf. at Missoula, MT, August 1989.

[DJS] G. David, J.-L. Journe, and S. Semmes, Operateurs de Calderon-Zygmund, fonction paraaccretive et interpolation, Rev. Mat. Iberoamericana 1 (1985), 1-56.

[HS] Y. Han and E. Sawyer, Littlewood-Paley theory on spaces of homogenous type and the classical function spaces, Mem. Amer. Math. Soc. 110 (1994), 1-136.

[M] Y. Meyer, Les nouveaux operateurs de Calderon-Zygmund, Colloque en l'honneur de $\mathrm{L}$. Schwartz, 1, Asterisque 131 (1985), 237-254.

[MS] R. A. Macias and C. Segovia, Lipschitz function on spaces of homogeneous type, Adv. Math. 33 (1979), 257-270.

[P] J. Peetre, New thoughts on Besov spaces, Duke Univ. Press, Durham, 1976.

[T] H. Triebel, Theory of function spaces, Birkhauser-Verlag, Berlin, 1983.

Department of Mathematics, Auburn University, Auburn, Alabama 36849-5310

E-mail address: hanyong@mail auburn.edu 